\title{
Pregnancy with Unknown Primary Cancer. A Rare Occurrence
}

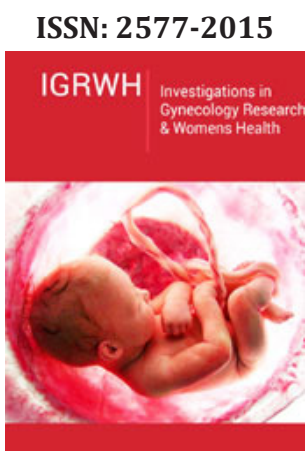

*Corresponding author: Ahuja M, Department of Obstetrics and Gynecology, School of Medical Sciences and Research, India

Submission: 監 June 11, 2019

Published: 筁July 09, 2018

Volume 3 - Issue 1

How to cite this article: Ahuja M, Yadav V, Jamal S, Goel N, Chutani N. Pregnancy with Unknown Primary Cancer. A Rare Occurrence. Invest Gynecol Res Women's Health.3(1). IGRWH.000553.2019.

DOI: 10.31031/IGRWH.2019.03.000553

Copyright@Ahuja M, This article is distributed under the terms of the Creative Commons Attribution 4.0 International License, which permits unrestricted use and redistribution provided that the original author and source are credited.

\author{
Ahuja M*, Yadav V, Jamal S, Goel N and Chutani N \\ Department of Obstetrics and Gynecology, School of Medical Sciences and Research, India
}

\begin{abstract}
Objective: Cancer of unknown primary in pregnancy is scarcely reported in literature. The mortality is very high and less than $25 \%$ survive beyond 1 year. The non-specific symptoms of pregnancy together with limited safe diagnostic modalities delays its early diagnosis hence resulting in the advanced stage of presentation with fatal outcome.

Case report: We present a case of 30-year-old G3 A2 at 27 weeks amenorrhea with non-specific complaints of weakness of both lower limbs which on MRI revealed pathological fracture of T12 vertebra and a mass in the iliac region. Biopsy from the iliac mass revealed undifferentiated adenocarcinoma which suggested metastasis. Patient underwent LSCS at 30 weeks followed by spinal fixation for the pathological fracture on post-operative day 9. After two days of spinal fixation patient succumbed to cardiac arrest.
\end{abstract}

Conclusion: Due to its rarity with acute deadly outcome, the previous literature related to the patients of cancer of unknown primary in pregnancy was reviewed and their final outcomes were studied.

Keywords: CUP; Pregnancy; MRI

Abbreviations: CUP: Cancer of Unknown Pregnancy; MRI: Magnetic Resonance Imaging; PET: Positron Emission Tomography

\section{Introduction}

Cancer of unknown primary in pregnancy occurs in $3-5 \%$ of all population [1]. Because of its rare co-existence and deadly outcome both the diagnosis and the management become a challenge. The diagnosis is difficult due to non-specific symptoms, signs and limited availability of safe diagnostic modality during pregnancy. Fetus as a patient becomes one of the deciding factors but delaying delivery till the time of attaining fetal salvageability can jeopardize and compromise the mother's condition. We present a case of 30-year-old G3 A2 with non- specific symptoms of weakness and paresthesia of both lower limbs which on MRI revealed fracture of T12 vertebrae due to suspected secondaries. Biopsy from the iliac mass revealed adenocarcinoma suggestive of metastasis. All other investigations to find primary were fruitless. Patient underwent LSCS at 30 weeks gestation due to an hydramnios and unfavorable cervix and delivered a male baby boy of 1200 grams. Simultaneous chemo-port insertion was also done post LSCS. Patient underwent spinal fixation on day 9 but succumbed to cardiac arrest on day 14. The effects of the disease on mother and the fetus should be borne in mind, as well as the fact that the pregnancy itself may affect the diagnostic and therapeutic procedures. Most patients present at late stage in pregnancy because of lack of awareness, ignorance and delay in availability of appropriate expertise for diagnosing such a modality.

\section{Case Report}

A 30-year-old G3A2 with previous two spontaneous abortions presented to the causality with 27 weeks of gestation with weakness of both lower limbs since last 20 days. The severity of weakness resulted in her inability to walk. There was no history of fever or any injury endured. There were no associated urinary and bowel deficit. The only significant history was of pulmonary tuberculosis for which she had taken treatment 10 years back. On examination patient was thin built, not pale and vitals were normal. There was hyperesthesia of both lower limbs and the power of both the lower limbs was of grade 2/5. MRI revealed pathological fracture of T12 vertebra with suspected metastatic lesions on left iliac bone. There was another metastatic lesion of in the left lower chest. Bowel lumen, kidney and liver 
were normal on MRI. Biopsy from the iliac mass revealed poorly differentiated adenocarcinoma of uncertain primary. USG neck, breast, upper abdomen was normal. USG obstetrics revealed a live fetus of 28 weeks with parameters corresponding to gestational age with an hydramnios. Pap smear was negative for intraepithelial lesion. CA125, CA15-3, LDH levels were normal. After consultation with the medical and surgical oncologist it was decided to start chemotherapy post LSCS in order to avoid any harmful effects of the chemotherapeutic drugs. LSCS with simultaneous chemo-port insertion at the level of jugular vein was performed at 30 weeks and a male baby weighing 1200 grams was delivered. Intra-operatively the uterus with adnexa, undersurface of diaphragm, liver, spleen, small and large bowel were found normal. on palpation. The placenta on histopathology did not show any evidence of metastasis. Postoperative period was uneventful and therapeutic low molecular weight heparin was also given. On post-operative day 10 spinal fixation of fractured thoracic vertebrae was done. On day 12 patient developed severe respiratory distress for which patient was shifted to ICU. There were rales in the lungs. Supportive treatment with oxygen and broad-spectrum antibiotics was started. This distress was followed by a sudden cardiac arrest on day 14 post LSCS and even after resuscitation patient could not be revived.

\section{Discussion}

Cancer in pregnancy occurs at the rate of 1 in 1000 . The most common malignancy associated with pregnancy is breast followed by cervix, lymphoma, leukemia and malignant melanoma. There are currently insufficient data areas in the literature regarding stage 4 presenting in pregnancy for the first time. The presentation is non-specific ranging from neurological deficit to neck masses. The case presented with weakness of both the lower limbs. Cancer of unknown primary can present as metastasis to the lymph node (67\%), liver (44\%), bone (39\%), lungs $(28 \%)$ followed by peritoneum and subcutaneous tissue (17\%) [2]. Our patient presented with bone metastasis. Bone metastasis usually involves T11 vertebrae [3]. Malignant melanoma usually metastasizes to the placenta but adenocarcinoma spilling to the placenta also has been reported [4]. It has been documented that placental metastasis can cause abruption and henceforth intrauterine death [5]. Along with placental metastasis fetal metastases have also documented [6]. In the present case histopathology of the placenta did not show any metastasis. MRI and ultrasound are the only two modalities found safe in pregnancy. PET scan is contraindicated in pregnancy. MRI in our case revealed suspected metastatic secondaries in the bone causing pathological fracture. The one differential diagnosis of this condition is tuberculosis; hence biopsy is mandatory for its confirmation. Starting chemotherapy during pregnancy carries many ethical issues. The fetus as a patient must be considered.

Chemotherapy is important for advanced stage, however contraindicated during first stage. Platinum based chemotherapeutic drugs are considered safe during second and third trimester but long-term outcomes of children exposed in utero to such chemotherapeutic drugs is poorly documented. In early gestation with cancer of unknown primary, the pregnancy can be terminated but in fetuses of near lung maturity the decision as to decide the ideal time and mode for delivery is a challenge for both the obstetrician as well as the neonatologist. LSCS was performed at 30 weeks of gestation after steroid coverage due to an hydramnios, extreme prematurity, unfavorable cervix combined with extreme weakness of both the lower limbs. The mode of delivery could either be vaginal or caesarean and this depends upon the BISHOP score and the overall general health of the patient. The chemo-port insertion in the internal jugular vein at the same setting of LSCS is the good practice point as it prevents multiple cannulation, avoids local complication such as superficial thrombophlebitis and ensures long term delivery of chemotherapeutic drugs. Spinal fixation as a mode of palliative treatment has good results although radiotherapy, vertebroplasty/kyphoplasty and steroid injections are other modalities [4]. The mean survival of such patients is not more than 2 years and it depends upon the histology of such metastasis. Generally, the terminal event is sudden which may be due to metastatic embolism or cardiac arrest as seen in the present case.

\section{Conclusion}

Cancer of unknown primary in pregnancy is a very rare coexistence. The acutecatastrophic outcome castsanimpressionable experience in the minds of the managing obstetrician. The management of such cases requires a multidisciplinary involvement of the obstetrician, medical and surgical oncologist, pathologist and the neonatologist.

\section{Conflict of Interest}

There is no disclosure of interest. No ethical issues were involved, and patient consent was taken.

\section{References}

1. Pavlidis N, Peccatori F, Fiona l, Anthony G (2015) Cancer of unknown primary during pregnancy: An exceptionally rare coexistence. Anticancer Res 35(1): 575-579.

2. Berghella V, Broth RE, Chapman AE, Cardonick E (2003) Metastatic unknown primary tumor presenting in pregnancy as multiple cerebral infracts. Obstet Gynecol 101(5): 1060-1062.

3. Jae HC, Jung KH, Choon HJ, Sung CL, Lee DH (2015) Patterns of treatment for metastatic pathological fractures of the spine: The efficacy of each treatment modality. Clin Orthop Surg 7(4): 467-482.

4. Gourley C, Monaghan H, Beattie G, Court S, Love C, et al. (2002) Intrauterine death resulting from placental metastases in adenocarcinoma of unknown primary. Clin Oncol (R Coll Radiol) 14(3): 213-216.

5. Momeni M, Cantu J, Young A (2013) Placental abruption and fetal demise secondary to placental metastasis from unknown primary: A case report. J Reprod Med 58(7-8): 341-343.

6. Pavilides N, Pentheroudakis G (2008) Metastatic involvement of placenta and foetus in pregnant women with cancer. Recent Results Cancer Res 178: $183-194$ 